\title{
Avaliação das concepções de educadores de escolas públicas e particulares sobre uso de drogas: um estudo exploratório
}

\section{Evaluation of teachers' conceptions about substance misuse in public and private schools: an exploratory}

Evaluación de concepciones de los educadores de escuelas públicas y particulares sobre el uso de drogas: un estudio exploratorio

\author{
Fernando Baptista de Souza* \\ Universidade Federal de São Paulo - Unifesp, São Paulo, São Paulo, Brasil
}

\author{
André Luiz Monezi Andrade** \\ Universidade Federal de São Paulo - Unifesp, São Paulo, São Paulo, Brasil
}

Thiago Pavin Rodrigues***

Universidade Federal de São Paulo - Unifesp, São Paulo, São Paulo, Brasil

\section{Marcelo Oliveira do Nascimento****}

Universidade Federal de São Paulo - Unifesp, São Paulo, São Paulo, Brasil

\section{Denise De Micheli*****}

Universidade Federal de São Paulo - Unifesp, São Paulo, São Paulo, Brasil

\begin{abstract}
RESUMO
O uso abusivo de drogas configura-se como um grave problema de saúde pública. O presente trabalho buscou identificar as principais crenças e conhecimentos de educadores sobre o uso de drogas. Participaram do estudo 105 educadores de escolas públicas e 399 de escolas particulares da cidade de São Paulo. A coleta de dados ocorreu a partir da aplicação de um questionário fechado, de autopreenchimento e anônimo. Aproximadamente $32 \%$ dos educadores de escolas públicas e $44 \%$ de escolas particulares afirmaram não ter conhecimento suficiente sobre o tema. Além disso, cerca de $50 \%$ dos educadores afirmaram ser a maconha a porta de entrada para o uso de outras drogas e $64 \%$ dos educadores de escolas públicas e $46 \%$ de escolas particulares concordaram que as drogas provocam apenas a ilusão do prazer. Os dados indicam a necessidade da implementação de programas de capacitação, desconstruindo crenças baseadas no senso comum e facilitando o acesso desse público a conteúdos científicos.

Palavras-chave: abuso de drogas, prevenção, professores, adolescente, educação.
\end{abstract}




\begin{abstract}
The substance abuse is considered a harmful healthy problem nowadays. This study aimed to identify the main educators' about substance misuse. Out of 105 educators from public school and 399 from private schools from the city of Sao Paulo participated of this study. All collected from a specific questionnaire developed by the authors. About 32\% of the educators from public school and $44 \%$ from private school assumed that not have enough information about substance misuse. Moreover, $50 \%$ of educators that marijuana is a gateway to the use of additional drugs and $64 \%$ from public schools and $46 \%$ from private schools believe that drugs leads only to the illusion of pleasure. These data suggest the necessity of a training program implementation to refresh on some beliefs based on a common sense and an access to scientific contents.
\end{abstract}

Keywords: substance misuse prevention, educators, adolescents, education.

\title{
RESUMEN
}

El presente estudio buscó identificar las concepciones fundamentales y los conocimientos de los educadores sobre el uso de drogas. Participaron 105 educadores de escuelas públicas y 399 de escuelas privadas de la ciudad de São Paulo. Los datos fueron colectados a partir de la aplicación de un cuestionario anónimo autoadministrado y cerrado. Aproximadamente $32 \%$ de los educadores de escuelas públicas y el $44 \%$ de escuelas particulares afirmaran que no tenían suficiente conocimiento sobre el tema. Además, acerca del $50 \%$ de todos los educadores, afirmaran que la marihuana es una puerta de entrada para otras drogas y el $64 \%$ de los educadores de escuelas públicas y el $46 \%$ de escuelas particulares están de acuerdo en que las drogas causan sólo una ilusión de placer. Los datos indican la necesidad de implementar programas de capacitación, descentrando creencias basadas en sentido común y facilitando el acceso al contenido de base científica.

Palabras-clave: abuso de drogas, prevención, profesores, adolescentes, educación.

\section{I ntrodução}

A adolescência é uma fase onde ocorrem diversas mudanças biopsicossociais, resultantes da interação constante entre processos psicobiológicos de desenvolvimento. É um período fortemente influenciado por variáveis socioeconômicas e a normas e valores culturais específicos (Ernst, Pine, \& Hardin, 2006). Nessa fase, em geral, o adolescente sente necessidade de vivenciar novas sensações e experiências, caracterizando a adolescência como "fase da experimentação" (Geier, 2013). Outra característica marcante deste período é a influência exercida pelo grupo de amigos, os quais podem, muitas vezes, favorecer o início do uso de substâncias. Isso ocorre devido a necessidade do adolescente em sentir-se aceito por seus pares (Gross et al., 2014).

Atualmente, o uso abusivo e a dependência de drogas configuram-se como um grave problema de saúde pública. De acordo com um relatório publicado em 2014 pelo Escritório das Nações Unidas Contra 
Drogas e Crime (UNODC, 2014), cerca de 243 milhões de indivíduos ( $5 \%$ da população global) já fizeram uso na vida de alguma substância ilícita. Destes, aproximadamente 27 milhões possuem problemas mais graves, com destaque para o uso da maconha, seguida de cocaína e anfetaminas. No Brasil, de acordo com um levantamento sobre o uso de drogas por estudantes brasileiros de ensino fundamental e médio, $25 \%$ relataram ter feito uso de cocaína nos últimos 12 meses (Carlini et al., 2012). Entre universitários, 49\% já experimentaram alguma substância ilícita e 80\% consumiram álcool em algum momento da vida de álcool, antes dos 18 anos (Oliveira, Alberghini, Santos, \& Andrade, 2013). Estes dados evidenciam que o consumo, bem como problemas decorrentes deste, podem atingir diferentes esferas da sociedade e diferentes faixas etárias. Em estudo realizado por Pechansky et al., (2009), foi verificado que estudantes que ingeriram uma dose de álcool apresentavam quatro vezes mais chance de pegar carona com motorista alcoolizado e aqueles que reportaram utilizar mais de cinco doses apresentaram uma chance cinco vezes maior de se envolverem em acidente automobilístico. Além disso, o consumo de drogas em idade inicial pode trazer prejuízos irreversíveis, em especial no que se refere a aspectos cognitivos (Andrade \& De Micheli, 2013).

Alguns autores também identificaram associação entre a precocidade de uso e o desenvolvimento de dependência (De Micheli \& Formigoni, 2004; Ernst, 2014). Esses achados são particularmente importantes uma vez que o processo de maturação neural pode ser um fator de risco para o consumo de drogas. Além disso, estressores psicossociais também podem favorecer 0 consumo de drogas na adolescência (Frade, De Micheli, Andrade \& Souza-Formigoni, 2013).

Assim, novos programas de prevenção precisam ser desenvolvidos em diferentes contextos e com a participação genuína de diversos profissionais (Moreira, Vóvio , \& De Micheli, 2015). A Organização Mundial de Saúde incentiva a proteção à saúde da população por meio da prevenção ao uso indevido de álcool e/ou outras drogas como medida prioritária nas políticas de saúde em geral (WHO, 2007). No entanto, observa-se que, apesar das prioridades destacadas em uma lista de iniciativas, no Brasil são desenvolvidas apenas campanhas discretas de conscientização do uso de álcool, evidenciando uma lacuna no desenvolvimento de programas escolares que contemplem especialmente os adolescentes (WHO, 2010). Segundo alguns autores, um dos ambientes mais propícios e indicados para o desenvolvimento de programas preventivos direcionados a adolescentes, é a escola (Babor \& Higgins-Biddle, 2000; Wagner, Tubman, \& Gil, 2004; Nascimento \& De Micheli, 2015). O ambiente escolar além de ser um espaço que propicia discussões e elaboração de estratégias de prevenção, também possibilita o envolvimento de alunos, pais, educadores e a 
comunidade em geral (Foxcroft \& Tsertsvadze, 2011). Segundo a Política Nacional de Educação Permanente em Saúde (Ministério da Saúde, 2009), estão previstos o desenvolvimento e a integração de capacitações em várias áreas da saúde com diferentes perfis de profissionais. Contudo, apesar das evidências indicando ser a escola o espaço ideal para a implementação de programas preventivos, um fator limitante para o estabelecimento destes programas no contexto escolar é a deficitária preparação dos educadores para abordar esta temática junto aos alunos (Rohrbach, Graham, \& Hansen, 1993). Harthun, Dustman, Reeves, Hecht, e Marsiglia (2008) observaram a falta de suporte dos educadores, evidenciando a necessidade de projetos que visem desconstruir conceitos inadequados e preconceituosos relativos ao consumo de drogas e aos usuários de modo geral.

Desta forma, outros autores observaram em educadores representações sociais relacionadas aos aspectos negativos $\mathrm{e}$ estigmatizantes, como os sentimentos de impotência e medo, prejudicando a realização de ações preventivas eficazes (Araldi, Njaine, Oliveira, \& Ghizoni, 2012; Moreira, Vóvio, \& De Micheli, 2015). De modo a ampliar a compreensão sobre prevenção do uso de drogas no ambiente escolar, faz-se necessário conhecer as compreensões dos educadores sobre este tema, que em muitos casos é construída com base em mitos, crenças, valores, sentimentos e a visão de mundo que possuem. O presente estudo teve por objetivo identificar as principais concepções, conhecimentos e conceitos dos educadores de escolas da rede pública e particular da cidade de São Paulo em relação ao uso de drogas na adolescência, como forma de contribuir para a formação de educadores nos cursos de licenciatura bem como na formação continuada.

\section{Método}

\subsection{Participantes}

O estudo foi realizado com 505 educadores, sendo 106 de escolas públicas e 399 de escolas particulares da cidade de São Paulo. Os participantes representavam diversas categorias profissionais na escola (diretores, coordenadores pedagógicos, psicólogos, pedagogos, orientadores educacionais, professores da Educação Infantil (EI), Ensino Fundamental I (EFI), Ensino Fundamental II (EFII) e do Ensino Médio (EM). Os docentes do EF II e do EM representavam todas as áreas do conhecimento (humanas, exatas e biológicas). A seleção dos educadores ocorreu por uma amostra de conveniência. 


\subsection{Instrumento}

Elaborou-se um instrumento específico para a coleta dos dados sociodemográficos e das concepções sobre uso de drogas. O instrumento de autopreenchimento e anônimo, foi composto por vinte e quatro questões de múltipla escolha utilizando a metodologia da Escala Likert. Antes da aplicação, realizou-se um estudo prévio (piloto) com 12 profissionais de um curso de Pós-Graduação em Educação objetivando verificar a inteligibilidade e aceitabilidade do instrumento. A partir dos resultados encontrados, o instrumento foi ajustado e aperfeiçoado para sua versão final (em anexo).

\subsection{Procedimentos}

As escolas foram selecionadas com base no critério de conveniência e posteriormente contatadas por e-mail e/ou telefone. Após o contato inicial, agendou-se uma data para apresentação dos objetivos do estudo e formalização da autorização da Direção da Escola para a coleta de dados. Na sequência, foram levados às escolas selecionadas envelopes individuais lacrados contendo os questionários, bem como uma urna para o depósito dos mesmos pelos educadores, preservando o sigilo e o anonimato das informações. As urnas, localizadas na sala dos professores, foram retiradas após 30 dias do início do estudo. Ao final do estudo, também foram agendadas devolutivas em dias e horários específicos, previamente informados aos educadores pela direção das escolas.

\subsection{Análise dos dados}

Os dados foram digitados a partir do método de dupla digitação objetivando reduzir a margem de erro. Nenhum questionário foi excluído. Realizou-se análises descritivas para apresentar o perfil sociodemográfico dos participantes da pesquisa e testes estatísticos inferenciais não-paramétricos para o cruzamento entre algumas variáveis. Para verificar a associação entre as variáveis categóricas utilizou-se Testes de Qui-quadrado e Teste Exato de Fisher. Em todos os testes adotou-se o nível de significância de 5\%. O Software Estatístico utilizado para as análises foi o SPSS 20.0.

\section{5 Ética}

O estudo foi submetido e aprovado pelo Comitê de Ética e Pesquisa da Universidade Federal de São Paulo (CEP 1864/11). 


\section{Resultados}

A média de idade dos educadores foi de 42,5 anos ( $\pm 10,6$ anos). Em relação à carga horária de trabalho, observou-se uma média de trabalho semanal de 25,9 horas $( \pm 10,7)$ sendo que a maior parte concentrava-se nos períodos matutino (69\%) e vespertino (63\%). Houve predominância do sexo feminino (77\%). Em relação a categoria profissional, a maioria era professores $(87 \%)$ desde as séries iniciais do Ensino Infantil (El) até o Ensino Médio (EM). Cerca de um terço eram professores do EM e ministravam disciplinas da área de humanas.

Dentre os principais resultados, evidenciamos a crença dos educadores de que a Maconha é a porta de entrada para o uso de outras drogas, e também a não associação entre 0 prazer proporcionado pelas drogas e o seu uso, como descrito na Tabela 1. Além disso, detectou-se diferença significativa para as seguintes afirmações: "As drogas provocam, apenas, a ilusão de prazer" $\left(x^{2}=11,067 ; \quad p=0,004\right)$; "As drogas não provocam prazer" $\left(x^{2}=13,012 ; p=0,001\right)$; "O uso de drogas pode ser um problema de falta de caráter" $\left(x^{2}=6,594 ; p=0,037\right)$ e "O uso de drogas pode ser causado por problemas psicológicos como ansiedade, insatisfação, depressão, insegurança, fuga de problemas, dentre outros" $\left(x^{2}=9,306 ; p=0,010\right)$. 
Fernando Baptista de Souza, André Luiz Monezi Andrade, Thiago Pavin Rodrigues, Marcelo Oliveira do Nascimento, Denise De Micheli

Tabela 1

Comparações das crenças dos educadores relacionadas a questões de caráter moral e conceituais, de ambas as redes de ensino (pública e privada) em relação ao uso e abuso de drogas $(n=505)$. ${ }^{*} p<0,05$.

\begin{tabular}{|c|c|c|c|c|c|c|}
\hline & Escola & Discordo & Indeciso & Concordo & $\mathbf{x}^{2}$ & $p$ \\
\hline \multirow{2}{*}{$\begin{array}{l}\text { O álcool é uma } \\
\text { droga lícita } \\
\text { porque não } \\
\text { causa muitos } \\
\text { prejuízos à } \\
\text { saúde. }\end{array}$} & Pública & 89 & 4 & 7 & 2,24 & 0,32 \\
\hline & Privada & 85 & 3 & 12 & & \\
\hline \multirow{2}{*}{$\begin{array}{l}\text { A maconha é a } \\
\text { porta de } \\
\text { entrada para o } \\
\text { uso de outras } \\
\text { drogas. }\end{array}$} & Pública & 20 & 8 & 72 & 2,67 & 0,26 \\
\hline & Privada & 19 & 15 & 66 & & \\
\hline \multirow{2}{*}{$\begin{array}{l}\text { As drogas } \\
\text { provocam, } \\
\text { apenas, a } \\
\text { ilusão de } \\
\text { prazer. }\end{array}$} & Pública & 25 & 11 & 64* & 11,06 & 0,00 * \\
\hline & Privada & $31^{*}$ & $22^{*}$ & 46 & & \\
\hline \multirow{3}{*}{$\begin{array}{l}\text { As drogas năo } \\
\text { provocam } \\
\text { prazer. }\end{array}$} & Pública & 67 & 14 & $19 *$ & 13,01 & $0,00 *$ \\
\hline & Privada & $77^{*}$ & $16^{*}$ & 7 & & \\
\hline & Privada & 64 & 16 & 20 & & \\
\hline \multirow{3}{*}{$\begin{array}{l}\text { Criaçăo e } \\
\text { ambiente } \\
\text { familiar podem } \\
\text { favorecer a } \\
\text { dependência }\end{array}$} & Pública & 15 & 4 & 81 & 3,42 & 0,18 \\
\hline & Privada & 9 & 6 & 85 & & \\
\hline & & & & & & \\
\hline \multirow{2}{*}{$\begin{array}{l}\text { Amigos podem } \\
\text { influenciar no } \\
\text { uso de drogas }\end{array}$} & Pública & 8 & 1 & 91 & 4,13 & 0,12 \\
\hline & Privada & 4 & 1 & 95 & & \\
\hline \multirow{3}{*}{$\begin{array}{l}\text { O uso de } \\
\text { drogas pode } \\
\text { ser um } \\
\text { problema de } \\
\text { falta de } \\
\text { caráter. }\end{array}$} & Pública & 75 & $12^{*}$ & $13^{*}$ & 6,59 & $0,03^{*}$ \\
\hline & Privada & $85^{*}$ & 7 & 8 & & \\
\hline & & & & & & \\
\hline \multirow{3}{*}{$\begin{array}{l}\text { O uso de } \\
\text { drogas pode } \\
\text { ser causado } \\
\text { por problemas } \\
\text { psicológicos } \\
\text { como } \\
\text { ansiedade, } \\
\text { insatisfação, } \\
\text { depressão, } \\
\text { insegurança, } \\
\text { fuga de } \\
\text { problemas, e } \\
\text { outros. }\end{array}$} & Pública & $12^{*}$ & 1 & 87 & 9,30 & 0,01 * \\
\hline & Privada & 5 & $3^{*}$ & $92 *$ & & \\
\hline & & & & & & \\
\hline
\end{tabular}


Quando questionada a forma de se abordar o tema em sala de aula, os educadores relatam que o tema deva ser tratado sem preconceito, porém dizem ser importante ressaltar os aspectos negativos causados pelas drogas. Assim, detectou-se diferença significativa para a seguinte afirmação "Não se sente à vontade em abordá-lo, pois não tem conhecimento sobre o tema" $\left(x^{2}=4,794 ; p=0,029\right)$ como descrito na Tabela 2.

\section{Tabela 2}

Comparação sobre a opinião dos educadores de ambas as redes de ensino (pública e privada) em relação a forma como deve ser abordado o tema em sala de aula, bem como seus conhecimentos relativos ao tema $(n=505)$. Porcentagem de concordancia com a afirmaçao. ${ }^{*} p<0,05 .{ }^{* *}$ Teste exato de Fisher.

\begin{tabular}{|c|c|c|c|c|}
\hline & $\begin{array}{c}\text { Tipo de } \\
\text { Escola }\end{array}$ & $\%$ & $\overline{X^{2}}$ & $p$ \\
\hline $\begin{array}{l}\text { Não acho aconselhável falar } \\
\text { sobre drogas. }\end{array}$ & $\begin{array}{l}\text { Pública } \\
\text { Privada }\end{array}$ & $\begin{array}{l}2 \\
1\end{array}$ & $0,196 * *$ & 0,153 \\
\hline $\begin{array}{l}\text { Deve ser conversado sobre } \\
\text { todas as drogas de uma } \\
\text { forma aberta e } \\
\text { preconceitos. }\end{array}$ & $\begin{array}{l}\text { Pública } \\
\text { Privada }\end{array}$ & $\begin{array}{l}70 \\
78\end{array}$ & 2,856 & 0,091 \\
\hline $\begin{array}{l}\text { E importante ressaltar os } \\
\text { malefícios provocados pelas } \\
\text { drogas, de modo a inibir a } \\
\text { experimentação e/ou o } \\
\text { consumo. }\end{array}$ & Pública & 35 & 0,103 & 0,748 \\
\hline $\begin{array}{l}\text { Não me sinto à vontade em } \\
\text { abordar o tema, devido à } \\
\text { falta de conhecimento. }\end{array}$ & $\begin{array}{l}\text { Pública } \\
\text { Privada }\end{array}$ & $\begin{array}{c}32 \\
44^{*}\end{array}$ & 4,794 & $0,029 *$ \\
\hline $\begin{array}{l}\text { Sinto-me a vontade para } \\
\text { abordar o assunto durante as } \\
\text { aulas, pois acredito ter } \\
\text { conhecimento }\end{array}$ & $\begin{array}{l}\text { Pública } \\
\text { Privada }\end{array}$ & 22 & 2,589 & 0,108 \\
\hline
\end{tabular}

\section{Discussão}

Neste estudo, observou-se que os educadores acreditam ser importante falar sobre drogas, embora percebam como insuficiente 0 conhecimento que possuem sobre o tema (32\% dos educadores de escolas públicas e $44 \%$ de escolas particulares), além destes conhecimentos serem pautados no senso comum. Com base nestes achados, observou-se disposição dos educares para serem treinados, compreendendo o quão importante é sua participação no processo de prevenção. Em estudo realizado por Nascimento, Vitalle e De Micheli (2012) $44 \%$ dos professores relataram fazer leituras superficiais 
sobre o assunto, o que é considerado muito distante do intuito de trabalhar a prevenção de drogas junto aos alunos (Ferreira, Sanchez, Ribeiro, Oliveira, \& Nappo, 2010). Em outro estudo realizado com educadores de escolas públicas e particulares, a maioria afirmou não ter informação suficiente sobre drogas, para transmiti-las aos alunos. Além disso, no referido estudo, os professores demonstram insegurança para fazer prevenção ao uso de drogas, justificando tal atitude à falta de informação e ao receio de não corresponderem às expectativas dos dos estudantes.

Neste sentido, estudos indicam que a deficitária formação dos educadores representa um dos fatores que dificulta a implantação de programas preventivos nas escolas (Harthun et al., 2008). Na Política Nacional de Educação Permanente em Saúde existem diretrizes bem estabelecidas que proporcionam o desenvolvimento de novas competências a partir de treinamentos (extensão continuada) para diversos profissionais, dentre eles, os educadores (Ministério da Saúde, 2009). Essa iniciativa tem por objetivo uma gerência descentralizada, de modo que o educador possa atuar enquanto um agente de promoção e prevenção em saúde no contexto escolar, fortalecendo a política de prevenção de drogas no contexto da educação. Em alguns estudos é bem estabelecido que o educador representa um modelo de identificação para o adolescente, podendo contribuir no processo de formação destes (Abry, Rimm-Kaufman, Larsen, \& Brewer, 2013).

Como vimos, se por um lado muitos professores não se sentem suficientemente preparados para fazer prevenção, por outro, muitos coordenadores educacionais julgam adequado e suficiente o preparo dos professores para tal atividade, numa ordem de 59\% segundo o estudo de Moreira, Silveira e Andreoli (2006). Podemos inferir que essa percepção paradoxal entre ambas as partes, deva-se a posição distanciada do coordenador, no que se refere a rotina interna da sala de aula impossibilitando-o de perceber o fenômeno tal como ele se apresenta.

No que se refere a decisão sobre consumir, ou não, álcool e/ou outras drogas, um estudo realizado por Sanchez, Oliveira, Ribeiro, \& Nappo, (2010) mostrou que a prevenção com base apenas no fornecimento de informações sobre as substâncias, não refreou a decisão em consumir. Segundo os referidos autores, quando solicitado aos estudantes que descrevessem o tipo de informações fornecidas na escola, muitos sequer recordavam. Além disso, estudo avaliando o método de prevenção baseado em informações, alerta para o cuidado que se deve ter a medida em que informações incompletas ou sem embasamento científico podem despertar o interesse dos estudantes pela experimentação de drogas (Nascimento, De Micheli, \& Vitalle, 2012). 
No presente estudo, quando analisado a opinião dos professores sobre a forma em que se deve abordar o tema drogas em sala de aula, $70 \%$ dos educadores de escolas públicas e $78 \%$ de escolas particulares consideraram que este deve ser tratado de forma sincera e livre de preconceitos. Entretanto, em outra questão, observou-se que $35 \%$ dos educadores de escolas públicas e $37 \%$ de escolas particulares afirmaram a importância em se ressaltar os aspectos negativos, por acreditar que deste modo o adolescente ficará temeroso em usar drogas. Estes achados indicam certa contradição, pois ao mesmo tempo em que acreditam que o assunto deva ser tratado sem preconceitos e abertamente, referem a importância em ressaltar os aspectos negativos do uso de substâncias. Esta incongruência no posicionamento e postura dos educadores, talvez deva-se pela falta de treinamento adequado. De maneira similar, Cavalcante et al. (2005) ao questionarem um grupo de educadores sobre os malefícios causados pelas drogas, observaram que a maior parte deles atribuiu danos, alterações e prejuízos irreversíveis aos usuários, reforçando o aspecto negativo destas substâncias. Além disso, os participantes daquele estudo apresentavam expressões enfáticas, pejorativas e muitas vezes alarmistas. Segundo alguns autores, existe um pensamento generalizado de que os usuários de substâncias compõem um conjunto de pessoas e hábitos homogêneos, tomando-se como base um padrão criado pelos não usuários (Palamar, 2013).

Quando questionados se o álcool é uma droga lícita, por não causar prejuízos à saúde, apenas $7 \%$ dos educadores de escolas públicas e $12 \%$ de escolas particulares, do presente estudo, concordaram com essa afirmação. Este dado corrobora a literatura que afirma que, em geral, as pessoas consideram as drogas lícitas menos perigosas que as ilícitas, atribuindo à esta última um maior potencial deletério ao organismo (Tsering, Pal, \& Dasgupta, 2010). No entanto, vale destacar que esta é uma interpretação distorcida, pois, embora o álcool e o tabaco sejam substâncias lícitas, são as que mais causam problemas de saúde a nível global.

Quando questionados sobre o consumo de maconha favorecer o uso de outras substâncias mais pesadas, observou-se uma concordância da ordem de $50 \%$ dos educadores do presente estudo. Esses achados estão alinhados ao estudo de Moreira (2005) em que o autor observou uma concordância de $51 \%$ para esta afirmação. Além disso, esses dados indicam ser esta uma crença, parte do senso comum, uma vez que grande parte da população não considera o álcool e tabaco como drogas, apesar de apresentarem um conhecimento mínimo a respeito de seus prejuízos. Vale ressaltar que estudos epidemiológicos evidenciam que algumas substâncias lícitas como o álcool e ilícitas como a maconha possuem um forte papel como precursoras na trajetória de uso de outras substâncias de abuso 
(Quek et al., 2013). Além disso, há de se mencionar o papel da mídia nesse processo de construção e fomentação de mitos e crenças, uma vez que estas agências priorizam a ênfase nas drogas ilícitas, frequentemente sobrepondo-as às lícitas, fortalecendo coletivamente a ideia de que são mais prejudiciais e destruidoras (Noto et al., 2003).

Neste estudo, observou-se um percentual significativamente maior de educadores de escolas públicas ( $19 \%$ vs. $7 \%$ de escolas particulares), afirmando que as drogas não provocam prazer, sendo esta sensação meramente ilusória. Poderíamos especular que talvez essa diferença deva-se ao fato de que muitos professores de escolas particulares têm mais acesso a informações baseadas em evidências científicas enquanto que os de escolas públicas possuem um acesso mais restrito a esse conteúdo.

Também, no presente estudo, observou-se um elevado percentual de educadores que mencionaram concordar que determinadas situações representam fator de risco para o consumo de álcool e/ou outras drogas por adolescentes, a saber: ambiente familiar desestruturado, conviver com amigos que usam drogas e presença de problemas psicológicos. Resultados semelhantes foram obtidos em outros estudos que mencionaram que $90 \%$ dos educadores acreditavam que viver num ambiente familiar conflituoso representava um fator de risco para o uso de drogas na adolescência, $72 \%$ mencionaram as amizades como fator de risco e $23 \%$ relacionavam o consumo de substâncias à presença de problemas psicológicos (Moreira et al., 2006). Semelhantemente, em um estudo realizado com professores portugueses, $18 \%$ apontaram ser 0 grupo de amigos um dos principais fatores de risco para o uso de drogas entre adolescentes (Gonçalves, 2008).

O presente estudo possui algumas limitações, entre elas o tamanho da amostra, que não nos permite a generalização dos dados encontrados. . Além disso, o estudo não apresenta metodologia de amostra aleatorizada e controlada de modo que a extrapolação dos dados deve ser feita com cautela.

Em suma, observou-se que embora os educadores apresentem muitas concepções sem embasamento científico e não se sintam seguros sobre abordar esta temática junto aos estudantes, a maioria se mostrou disposto e motivado para participar de cursos e programas de capacitação. Assim, a implementação de programas de capacitação direcionados a educadores, pautados numa proposta abrangente e continua, é essencial para a formação de agentes engajados genuinamente em políticas de prevenção e promoção de saúde, favorecendo o avanço no processo de transformação da realidade social em que atuam. 


\section{Referências}

Abry, T., Rimm-Kaufman, S. E., Larsen, R. A., \& Brewer, A. J. (2013). The influence of fidelity of implementation on teacher-student interaction quality in the context of a randomized controlled trial of the Responsive Classroom approach. J Sch Psychol, 51(4), 437-453.

Andrade, A. L. M. , \& De Micheli, D. (2013). Cognitive Aspects of Fetal Alcohol Syndrome in Young Adults: Two Case Studies. Interação em Psicologia, 17(2), 217-223.

Araldi, J. C., Njaine, K., Oliveira, M. C., \& Ghizoni, A. C. (2012). Representações sociais de professores sobre o uso abusivo de álcool e outras drogas na adolescência: repercussões nas ações de prevenção na escola. Interface - Comunicação, Saúde, Educação, 16(40), 135-146.

Babor, T. F., \& Higgins-Biddle, J. C. (2000). Alcohol screening and brief intervention: dissemination strategies for medical practice and public health. Addiction, 95(5), 677-686.

Carlini, E. A., Noto, A. R., Sanchez, S. V., Carlini, C., Locatelli, D. P., Abeid, L., et al. (2012). VI Levantamento Nacional Sobre o Consumo de Drogas Psicotrópicas Entre Estudantes do Ensino Fundamental e Médio das Redes Pública e Privada de Ensino nas 27 Capitais Brasileiras. São Paulo: Centro Brasileiro de Informações Sobre Drogas Psicotrópicas - CEBRID.

De Micheli, D., \& Formigoni, M. L. O. S. (2004). Drug use by Brazilian students: associations with family, psychosocial, health, demographic and behavioral characteristics. Addiction, 99(5), 570-578.

Ernst, M., Pine, D. S., \& Hardin, M. (2006). Triadic model of the neurobiology ofmotivated behavior in adolescence. Psychological Medicine, 36(3), 299-312.

Ernst, M. (2014). The triadic model perspective for the study of adolescent motivated behavior. Brain Cogn, 89, 104-111.

Ferreira, T. C. D., Sanchez, Z. M., Ribeiro, L. A., Oliveira, L. G., \& Nappo, S. A. (2010). Percepções e atitudes de professores de escolas públicas e privadas perante o tema drogas. Interface Comunicação, Saúde, Educação, 14, 551-562.

Foxcroft, D. R., \& Tsertsvadze, A. (2011). Universal school-based prevention programs for alcohol misuse in young people. Cochrane Database Syst, 11(5), 1-123, CD009113.

Frade, I. F., De Micheli, D., Andrade, A. L. M., \& Souza-Formigoni, M. L. O. (2013). Relationship between Stress Symptoms and Drug use among Secondary Students. Spanish Journal of Psychology, 16, 1-12. 
Geier, C. F. (2013). Adolescent cognitive control and reward processing: implications for risk taking and substance use. Horm Behav, 64(2), 333-342.

Gonçalves, A. (2008). Álcool, tabaco e outras drogas: concepções de professores e alunos de ensino básico e secundário e análise de programas e manuais escolares. (Tese de Doutorado), Universidade do Minho Portugal.

Gross, C., Neumann, M., Kalkbrenner, M., Mick, I., Lachnit, A., Reichert, J., \& Zimmermann, U. S. (2014). A Retrospective Analysis of Psychosocial Risk Factors Modulating Adolescent Alcohol Binge Drinking. European Addiction Research, 20(6), 285-292.

Harthun, M. L., Dustman, P. A., Reeves, L. J., Hecht, M. L., \& Marsiglia, F. F. (2008). Culture in the classroom: developing teacher proficiency in delivering a culturally-grounded prevention curriculum. The Journal of Primary Prevention, 29(5), 435-454.

Moreira, A., Vóvio, C. L., \& De Micheli, D. (2015) Drug abuse prevention in school: challenges and possibilities for the role of the educator. Revista de Educação e Pesquisa, São Paulo, 41(1), 119-134.

Moreira, F. G., Da Silveira, D. X. , \& Andreoli, S. B. (2006). Situações relacionadas ao uso indevido de drogas nas escolas públicas da cidade de São Paulo. Revista de Saúde Pública, 40, 810-817.

Nascimento, M. O., \& De Micheli, D. (2015). Evaluation of different school-based preventive interventions for reducing the use of psychotropic substances among students: a randomized study. Ciência \& Saúde Coletiva, 20(8), 2499-2510.

Nascimento, M. O., Vitalle, M. S. S., \& De Micheli, D. (2012). A visão e temores dos educadores ante ao uso abusivo de substâncias psicoativas por adolescentes no ambiente escolar. Revista Magistro, 2(1), 16.

Noto, A. R, Galduróz, J. C. F., Nappo, S. A., Fonseca, A. M., Carlini, C. M. A., Moura, Y. G., \& Carlini, E.A. (2003). Levantamento nacional sobre uso de drogas entre crianças e adolescentes em situação de rua nas 27 capitais brasileiras. Secretaria Nacional de Polísitca Sobre Drogas (SENAD): Centro Brasileiro de Informações Sobre Drogas Psicotrópicas (CEBRID).

Oliveira, L. G., Alberghini, D. G., Santos, B., \& Andrade, A. G. (2013). Polydrug use among college students in Brazil: a nationwide survey. Revista Brasileira de Psiquiatria, 35(3), 221-230.

Palamar, J. J. (2013). An examination of beliefs and opinions about drug use in relation to personal stigmatization towards drug users. J Psychoactive Drugs, 45(5), 367-373.

Pechansky, F., De Boni, R., Diemen, L. V., Bumaguin, D., Pinsky, I., Zalesky, M., et al. (2009). Highly reported prevalence of 
drinking and driving in Brazil: data from the first representative household study. Revista Brasileira de Psiquiatria, 31(2), 125130.

Ministério da Saúde (2009). Política Nacional de Educação Permanente em Saúde. Departamento de Gestão da Educação em Saúde, Brasília.

Quek, L. H., Chan, G. C., White, A., Connor, J. P., Baker, P. J., Saunders, J. B., \& Kelly, A. B. (2013). Concurrent and simultaneous polydrug use: latent class analysis of an Australian nationally representative sample of young adults. Front Public Health, 1, 1-9.

Rohrbach, L. A., Graham, J. W., \& Hansen, W. B. (1993). Diffusion of a school-based substance abuse prevention program: predictors of program implementation. Preventive Medicine, 22(2), 237260.

Sanchez, Z. V. D. M., Oliveira, L. G., Ribeiro, L. A., \& Nappo, S. A. (2010). O papel da informação como medida preventiva ao uso de drogas entre jovens em situação de risco. Ciência \& Saúde Coletiva, 15, 699-708.

Tsering, D., Pal, R., \& Dasgupta, A. (2010). Substance use among adolescent high school students in India: A survey of knowledge, attitude, and opinion. Journal of Pharmacy and Bioallied Science, 2(2), 137-140.

UNODC. World Drug Report 2014. United Nations Office on Drugs and Crime - UNODC, 2014.Wagner, E. F., Tubman, J. G., \& Gil, A. G. (2004). Implementing school-based substance abuse interventions: methodological dilemmas and recommended solutions. Addiction, 99 Suppl 2, 106-119.

WHO. (2007). Who Expert Committee on Problems Related to Alcohol Consumption. Geneva: World Health Organization - WHO.

WHO. (2010). Global strategy to reduce the harmful use of alcohol. Geneva: World Health Organization.

\section{Endereço para correspondência}

\section{Fernando Baptista de Souza}

Universidade Federal de São Paulo

Unidade de Dependência de Drogas - Departamento de Psicobiologia

Rua Napoleão de Barros, 1038, Vila Clementino, CEP 04024-003, São Paulo - SP, Brasil

Endereço eletrônico: fernandobaptista_adr@yahoo.com.br

\section{André Luiz Monezi Andrade}

Universidade Federal de São Paulo

Unidade de Dependência de Drogas - Departamento de Psicobiologia

Rua Napoleão de Barros, 1038, Vila Clementino, CEP 04024-003, São Paulo - SP, Brasil

Endereço eletrônico: andremonezi@gmail.com

Thiago Pavin Rodrigues

Universidade Federal de São Paulo 
Fernando Baptista de Souza, André Luiz Monezi Andrade, Thiago Pavin Rodrigues, Marcelo Oliveira do Nascimento, Denise De Micheli

Unidade de Dependência de Drogas - Departamento de Psicobiologia

Rua Napoleão de Barros, 1038, Vila Clementino, CEP 04024-003, São Paulo - SP, Brasil

Endereço eletrônico: thiagopavin@gmail.com

Marcelo Oliveira do Nascimento

Universidade Federal de São Paulo

Unidade de Dependência de Drogas - Departamento de Psicobiologia

Rua Napoleão de Barros, 1038, Vila Clementino, CEP 04024-003, São Paulo - SP, Brasil

Endereço eletrônico: marcelozoologia@hotmail.com

\section{Denise De Micheli}

Universidade Federal de São Paulo

Unidade de Dependência de Drogas - Departamento de Psicobiologia

Rua Napoleão de Barros, 1038, Vila Clementino, CEP 04024-003, São Paulo - SP, Brasil

Endereço eletrônico: demicheli.unifesp@gmail.com

Recebido em: 14/08/2014

Reformulado em: 26/05/2015

Aceito para publicação em: 26/05/2015

\section{Notas}

* Mestre em Ciências pelo Programa de Pós Graduação em Educação e Saúde na Infância e Adolescência.

** Doutor em Psicobiologia. Docente do Departamento de Psicologia. Universidade Anhembi Morumbi - Laureate International Universities ${ }^{\circledR}$.

*** Mestre em Psicobiologia.

**** Mestre em Ciências pelo Programa de Pós Graduação em Educação e Saúde na Infância e Adolescência.

***** Pós Doutora em Ciências. Professora Adjunta do Departamento de Psicobiologia - UNIFESP. Unidade de Dependência de Drogas. 